The Nepali Math. Sc. Report, Vol. 38, No.2, 2021:35-45

DOI: $0.3126 /$ nmsr.v38i2.42708

\title{
A NUMERICAL SOLUTION OF WATER FLOW IN UNSATURATED SOIL WITH EVAPOTRANSPIRATION
}

\author{
RAMESH CHANDRA TIMSINA ${ }^{1}$, HARIHAR KHANAL ${ }^{2}$, ANDREI LUDU $^{2}$ AND \\ KEDAR NATH UPRETY ${ }^{3}$ \\ ${ }^{1}$ Department of Mathematics, Patan Multiple Campus, Tribhuvan University, \\ Kathmandu, Nepal \\ ${ }^{2}$ Department of Mathematics, Embry-Riddle Aeronautical University, Florida. U.S.A. \\ ${ }^{3}$ Central Department of Mathematics, Tribhuvan University, Kathmandu, Nepal
}

\begin{abstract}
Flow movement in unsaturated soil can be expressed by Richards equation. This equation can be obtained by applying the mass conversation law and the Darcy law. In this work, we solve one-dimensional Kirchhoff transformed Richards equation with loss of water due to the evaporation of unsaturated porous media (soils) and transpiration of plants numerically using Crank-Nicolson method. The result has compared with evapotranspiration function and without it in the governing equation. It has found that an additional work in time and flow movement is needs to reach the given boundary condition for the model without evapotranspiration.
\end{abstract}

Keywords: Finite Difference methods, Richards Equation, Kirchhoff Transformation, Infiltration Evapotranspiration.

\section{INTRODUCTION}

In many branches of science and engineering such as soil mechanics, fluid mechanics, agricultural engineering, environmental engineering and ground water hydrology, prediction of fluid movement in unsaturated porous media(soil) is an emerging problem. In unsaturated zone the fluid motion is assumed to obey the classical Richards equation, which is obtained by applying the mass conservation law and the Darcy law and can be expressed as different forms either pressure head $\psi$ or moisture content $\theta$ as the dependent variable. The constitutive relationship between $\theta=\theta(z, t)$ and $\psi=\psi(z, t)$ allows the conversion from one another where $t$ is the time, $z$ is the vertical dimension (downward positive). Equation (1.1) shows the coupled (mixed) form of Richards Equation[1].

$$
\frac{\partial \theta}{\partial t}-\nabla \cdot K(\psi) \nabla \psi-\frac{\partial K}{\partial z}=E T
$$

Where, $\theta$ is the volumetric moisture content, $\psi$ is the pressure head $K(\psi)$ is the unsaturated hydraulic conductivity, describes the ease with which water can move through pore space, and depends on the intrinsic permeability of the material and the properties of fluid such

Accepted/ Published Online: December, 2021 
as degree of saturation, density and the viscosity[2]. ET is the evapotranspiration and it represents the loss of water due to the evaporation of the unsaturated porous media (soils) and transpiration of plants and can be estimated by Penman-Monteith formula as [3]

$$
E T=\frac{0.408 \Delta\left(R_{n}-G\right)+\gamma \frac{900}{T+273} u_{2}\left(e_{s}-e_{a}\right)}{\Delta+\gamma\left(1+0.34 u_{2}\right)}
$$

Where ET: The reference evapotranspiration, $R_{n}$ : The net radiation at the crop surface, $G$ : The soil heat flux density, $T$ : The mean daily air temperature at $2 \mathrm{~m}$ height, $u_{2}$ : The wind speed at $2 \mathrm{~m}$ height, $e_{s}$ : The saturation vapor pressure, $e_{a}$ is actual vapor pressure, $e_{s}-e_{a}$ : The saturation vapor pressure deficit, $\Delta$ is slop vapor pressure curve and $\gamma$ : The psychometric constant.

Constitutive relations between $\theta=\theta(z, t)$ and $\psi=\psi(z, t)$ and the relation between $K$ and $\psi$ are developed appropriately, which consequently gives nonlinear behavior to equation (1.1). Reliable approximation of these relations are in general tedious to develop and thus also challenging. To gather the parameters either from field measurements or laboratory experiments are relatively expensive and such relations are limited to particular cases. Perhaps the most widely used empirical constitutive relations for the moisture content and hydraulic conductivity is due to the work of van Genuchten and Haverkamp et al.(particular case of van Genuchten). The following popular model from ground water hydrology due to Haverkamp et al[4], which describes these constitutive relation as the continuous function of $\psi$.

$$
\theta(\psi)=\theta_{r}+\frac{\alpha\left(\theta_{s}-\theta_{r}\right)}{\alpha+|\psi|^{\beta}}, K(\psi)=\frac{K_{s} A}{A+|\psi|^{\gamma}}
$$

where the subscript $s$ refer to the saturation i.e. the value of $\theta$ for which $\psi=0$ and the subscript $r$ to residual moisture content and $A, \alpha, \beta, \gamma$ are dimensionless soil parameters. Both the function $K(\psi)$ and $\theta(\psi)$ in equation 1.3 are highly nonlinear since they can be dramatically changed over a small range of $\psi$. With these the Richards equation (1.1) becomes a highly nonlinear partial differential equation and analytical solution is not possible except for some special cases.

The flow of water in unsaturated porous media(soil) is complex in nature. Because of its complexity and lack of absolute and reliable analytical solution to calculate the flow in unsaturated zone, the use of numerical methods to solve the problems related to unsaturated flow(Richards equation) has grown considerably in recent years. Since the expressions of equation (1.3) make the equation (1.1) highly nonlinear, it is important to utilize efficient and accurate solution procedures. Different numerical methods are used to approximate the solution of equation (1.1). Different methods yield different accuracy with computationally expensive and are not reliable for some cases. Therefore infiltration problems are still considered to be one of the most important topics of interest in groundwater hydrology. Many numerical schemes based on finite difference, finite element, finite volume and Adaptive time-stepping strategies have been developed to approximate the solution of the Richards equation [5], [6], [7], 8], 9], [10], [11].

Because of the strong stability restriction of the Forward Euler schemes for the parabolic partial differential equations, it seems there has not been much attention given to develop 
Forward Euler schemes for the Richards equation and adequate amount of research work is devoted in developing Backward Euler schemes. Due to the highly nonlinear nature of the problem, to implement Backward Euler schemes, no mater which numerical approaches we use, the problem has to be linearized somehow at some stage. As such some iterative approaches need to be applied to tackle this highly nonlinear problem [4, 12]. The Backward Euler schemes are unconditionally stable, but with the iterative process involved they all turn out to be computationally expensive and in certain circumstances, unreliable.

Contemporary research trends in computational approach is to develop efficient parallel algorithm for High-performing Computers. For parallelize the numerical procedures, the algorithm should be iteration free. For this, an Forward Euler scheme is the best option. In [13], a linearized Richards equation model is studied. In [9], stability analysis of Forward Euler scheme for the Richards equation is studied. In [14] an explicit stabilized RungeKutta- Legendre super time-stepping scheme for the Richards equation is studied. Here w Kirchhoff integral transform is used to reduce the highly nonlinear equation to a functional linear parabolic equation and solve it numerically.

The aim of this paper is to implement finite difference scheme(Crank-Nicolson) to Kirchhoff transformed Richards equation with evapotranspiration. The work here presented, describes and verifies the employment and accuracy of a Crank-Nicolson scheme to simulate flow in unsaturated porous media with evaporation of bare soils and transpiration of plants. The advantage of using our scheme is that it is unconditionally stable, easy to implement, can be easily extended to problems in higher dimensions, and can be easily parallelized.

This paper is organized as follows: In section 2, we present Kirchhoff transformation to (spatially) linearize the Richards equation. In section 3, we present the numerical methods based on finite difference method. In section 4, we compare the result with and without the sink terms in the Richards equation. Finally, in section 5 we present our conclusions.

\section{Simplified OnE-Dimensional RichaRds EQUATION}

Richards equation (1.1) in one space dimension with loss of water due to the evaporation of unsaturated porous media (soils) and transpiration of plants is considered as: .

$$
\frac{\partial \theta}{\partial t}=\frac{\partial}{\partial z}\left(K(\psi) \frac{\partial \psi}{\partial z}\right)-\frac{\partial K}{\partial z}+E T
$$

Generally Richards equation (2.1) is used to simulate infiltration experiments. These experiments begin with a wetting soil on top of the ground surface, showing a clear connection with the Darcy's law. Assuming that the infiltration with known pressure head at the top and bottom of the soil column. That is for the following initial and boundary conditions.

$$
\left\{\begin{array}{l}
\psi(z, 0)=\psi_{0}(z), 0<z<L \\
K(\psi)-K(\psi) \frac{\partial \psi}{\partial z}=q(t), z>0, t>0 \\
\psi(L, t)=\beta(t), t>0
\end{array}\right.
$$


2.1. Kirchhof integral transform. Applying Kirchhof integral transformation to equation (2.1), with $h=\psi-z$ (say) and define

$$
\phi(h)=\int_{0}^{h} \bar{K}(\lambda) d \lambda
$$

Since $K(h)>0$ from $(1.3)$, the function $\phi(h)$ is strictly increasing with $\bar{K}(h)=K(\psi)$. Taking derivative of both sides of the transformation, we obtain :

$$
\frac{\partial \phi}{\partial z}=\frac{\partial \phi}{\partial h} \frac{\partial h}{\partial z}=\bar{K}(h) \frac{\partial(\psi-z)}{\partial z}=K(\psi)\left(\frac{\partial \psi}{\partial z}-1\right)=K(\psi) \frac{\partial \psi}{\partial z}-K(\psi)
$$

Again taking derivative of equation 2.4,

$$
\frac{\partial^{2} \phi}{\partial z^{2}}=\frac{\partial\left(K(\psi) \frac{\partial \psi}{\partial z}\right)}{\partial z}-\frac{\partial}{\partial z}(K(\psi))
$$

Using the equation (2.5), the Richards equation 2.1 takes the form

$$
\frac{\partial \bar{\theta}}{\partial t}=\frac{\partial^{2} \phi}{\partial z^{2}}+E T
$$

with $\bar{\theta}(\phi)=\theta(h)$. The corresponding initial and boundary conditions for the transformed equation (2.6) takes the following form

$$
\left\{\begin{array}{l}
\phi(z, 0)=\phi_{0}(z), 0<z<L \\
\frac{\partial \phi}{\partial z}=\bar{q}(t), z>0, t>0 \\
\phi(L, t)=\bar{\beta}(t), t>0
\end{array}\right.
$$

The Kirchhoff transformation transformed the doubly nonlinear equation (2.1) to a nonlinear parabolic problem (2.6). Also noted that the Kirchhoff transformation preserves the uniqueness result for the transformed problem.

\section{Numerical Method}

To solve the equation (2.6) numerically with above mentioned initial and boundary conditions (2.7), it is more convenient to have a single state variable. For this, assuming $\theta$ and $\phi$ are single valued continuous functions of one another and rearrange to obtain

$$
\frac{\partial \theta}{\partial t}=\frac{\partial \theta}{\partial \phi} \frac{\partial \phi}{\partial t}=\left(\frac{1}{\frac{\partial \phi}{\partial \theta}}\right) \frac{\partial \phi}{\partial t}, \quad \frac{\partial \phi}{\partial \theta}=\frac{\partial \phi}{\partial h} \frac{\partial h}{\partial \theta}
$$

Differentiating (1.3) and (2.3) with respect to $h$

$$
\frac{\partial \theta}{\partial h}=\alpha\left(\theta_{s}-\theta_{r}\right)\left(\alpha+|h|^{\beta}\right)^{-2} \cdot \beta|h|^{\beta-1}, \quad \frac{\partial \phi}{\partial h}=\bar{K}(h)=K(\psi) .
$$

Using (3.1) and (3.2), the transformed Richards equation (2.6) takes the form:

$$
c(\phi) \frac{\partial \phi}{\partial t}=\frac{\partial^{2} \phi}{\partial z^{2}}+E T
$$

where the functional coefficient $c$ depends on $\phi$ through $h$ as

$$
c(\phi(h))=\frac{\alpha \beta\left(\theta_{s}-\theta_{r}\right)|h|^{\beta-1}}{\bar{K}(h)\left(\alpha+|h|^{\beta}\right)^{2}} .
$$


3.1. Finite difference discretization. Let $\Delta z=L / M$ and $\Delta t=T / N$. We construct a grid $\left(z_{j}, t_{n}\right)$, with $z_{j}=j \Delta z, j=0,1,2, \cdots, M$ and $t_{n}=n \Delta t, n=0,1,2, \cdots N$. Let $\phi_{j}^{n}$ denote $\phi\left(z_{j}, t_{n}\right)$. The partial differential equation (3.3) can be approximated using forward difference in time and central difference in space as

$$
\left.\frac{\partial \phi}{\partial t}\right|_{\left(z_{j}, t_{n}\right)} \approx \frac{\phi_{j}^{n+1}-\phi_{j}^{n}}{\Delta t},\left.\quad \frac{\partial^{2} \phi}{\partial z^{2}}\right|_{\left(z_{j}, t_{n}\right)} \approx \frac{\phi_{j-1}^{n}-2 \phi_{j}^{n}+\phi_{j+1}^{n}}{\Delta z^{2}}
$$

Let $0 \leq \lambda \leq 1$. Using a weighted average of the derivative $\frac{\partial^{2} \phi}{\partial z^{2}}$ at two time levels, $t_{n}$ and $t_{n+1}$, the equation $(3.3)$ can be discretized as:

$$
\phi_{j}^{n+1}=\phi_{j}^{n}+\sigma_{j}^{n}\left[\lambda\left(\phi_{j-1}^{n+1}-2 \phi_{j}^{n+1}+\phi_{j+1}^{n+1}\right)+(1-\lambda)\left(\phi_{j-1}^{n}-2 \phi_{j}^{n}+\phi_{j+1}^{n}\right)\right]+\sigma_{1 j}^{n}(E T)_{j}^{n}
$$

where $\sigma_{j}^{n}=\Delta t /\left(c_{j}^{n} \Delta z^{2}\right), \sigma_{1 j}^{n}=\Delta t / c_{j}^{n}$.

Equation $(3.6)$ is used to update the values of $\phi_{j}^{n+1}$ for the internal nodes. Using the second order central difference with ghost node approach at the upper boundary and constant pressure head at the lower boundary, we get

$$
\left\{\begin{aligned}
\left(1+2 \lambda \sigma_{j}^{n}\right) \phi_{0}^{n+1}-2 \lambda \sigma_{j}^{n} \phi_{1}^{n+1}= & {\left[1-2(1-\lambda) \sigma_{0}^{n}\right] \phi_{0}^{n}+} \\
2(1-\lambda) \sigma_{0}^{n} \phi_{1}^{n} & -2 \Delta z\left[\lambda \sigma_{0}^{n+1} q\left(t_{n+1}\right)+(1-\lambda) \sigma_{0}^{n} q\left(t_{n}\right)\right]+\sigma_{10}^{n}(E T)_{0}^{n} \\
\phi_{M}^{n+1}= & \bar{\beta}\left(t_{n+1}\right)
\end{aligned}\right.
$$

The numerical scheme (3.6)-3.7 represents a forward in time central in space (FTCS), backward in time central in space (BTCS) and Crank-Nicolson (CN) schemes for $\lambda=0, \lambda=$ 1 and $\lambda=\frac{1}{2}$ respectively [15]. The error associated with this approximation is $\mathcal{O}\left(\Delta z^{2}+\Delta t\right)$ for all $\lambda \neq \frac{1}{2}$. In the case of Crank-Nicolson, it is $\mathcal{O}\left(\Delta z^{2}+\Delta t^{2}\right)$, second order accurate in both space and time.

The above numerical procedure can be expressed in a tridiagonal matrix system as

$$
\left[\begin{array}{ccccccc}
1+2 \lambda \sigma_{0}^{n} & -2 \lambda \sigma_{0}^{n} & 0 & \ldots & 0 & 0 & 0 \\
-\lambda \sigma_{1}^{n} & 1+2 \lambda \sigma_{1}^{n} & -\lambda \sigma_{1}^{n} & \ldots & 0 & 0 & 0 \\
\ldots & \ldots & \ldots & \ldots & \ldots & \ldots & \ldots \\
\ldots & \ldots & \ldots & \ldots & \ldots & \ldots & \ldots \\
0 & 0 & 0 & \ldots & -\lambda \sigma_{M-1}^{n} & 1+2 \lambda \sigma_{M-1}^{n} & -\lambda \sigma_{M-1}^{n} \\
0 & 0 & 0 & \ldots & 0 & 0 & 1
\end{array}\right]\left[\begin{array}{c}
\phi_{0}^{n+1} \\
\phi_{1}^{n+1} \\
\ldots \\
\ldots \\
\phi_{M-1}^{n+1} \\
\phi_{M}^{n+1}
\end{array}\right]
$$




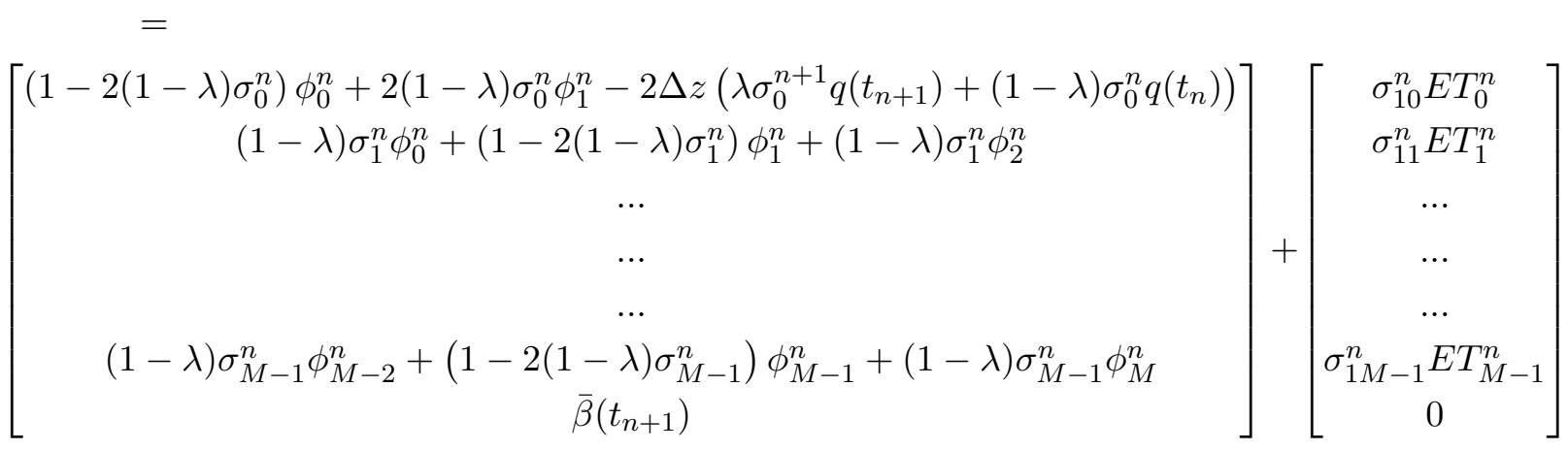

When $\lambda=\frac{1}{2}$ equation 3.8 takes the form

$$
\begin{aligned}
& {\left[\begin{array}{ccccccc}
1+\sigma_{0}^{n} & -\sigma_{0}^{n} & 0 & \ldots & 0 & 0 & 0 \\
-\frac{1}{2} \sigma_{1}^{n} & 1+\sigma_{1}^{n} & -\frac{1}{2} \sigma_{1}^{n} & \ldots & 0 & 0 & 0 \\
\ldots & \ldots & \ldots & \ldots & \ldots & \ldots & \ldots \\
\ldots & \ldots & \ldots & \ldots & \ldots & \ldots & \ldots \\
0 & 0 & 0 & \ldots & -\frac{1}{2} \sigma_{M-1}^{n} & 1+\sigma_{M-1}^{n} & -\frac{1}{2} \sigma_{M-1}^{n} \\
0 & 0 & 0 & \ldots & 0 & 0 & 1
\end{array}\right]\left[\begin{array}{c}
\phi_{0}^{n+1} \\
\phi_{1}^{n+1} \\
\ldots \\
\ldots \\
\phi_{M-1}^{n+1} \\
\phi_{M}^{n+1}
\end{array}\right]} \\
& \begin{array}{l}
= \\
{\left[\begin{array}{c}
\left(1-\sigma_{0}^{n}\right) \phi_{0}^{n}+\sigma_{0}^{n} \phi_{1}^{n}-\Delta z\left(\sigma_{0}^{n+1} q\left(t_{n+1}\right)+\sigma_{0}^{n} q\left(t_{n}\right)\right) \\
\frac{1}{2} \sigma_{1}^{n} \phi_{0}^{n}+\left(1-\sigma_{1}^{n}\right) \phi_{1}^{n}+\frac{1}{2} \sigma_{1}^{n} \phi_{2}^{n} \\
\cdots \\
\cdots \\
\cdots \\
\frac{1}{2} \sigma_{M-1}^{n} \phi_{M-2}^{n}+\left(1-\sigma_{M-1}^{n}\right) \phi_{M-1}^{n}+\frac{1}{2} \sigma_{M-1}^{n} \phi_{M}^{n} \\
\bar{\beta}\left(t_{n+1}\right)
\end{array}\right]+\left[\begin{array}{c}
\sigma_{10}^{n} E T_{0}^{n} \\
\sigma_{11}^{n} E T_{1}^{n} \\
\ldots \\
\cdots \\
\ldots \\
\sigma_{1 M-1}^{n} E T_{M-1}^{n} \\
0
\end{array}\right]}
\end{array}
\end{aligned}
$$

The numerical scheme $(3.9)$ can be used to update the transformed variable $\phi_{j}^{n}$ to its value in the next time level $\phi_{j}^{n+1}$. But it cannot advance the algorithm to the next time level $\phi_{j}^{n+2}$ without evaluating the function $c\left(\phi_{j}^{n+1}\right)$ which requires computing the intermediate variable $h_{j}^{n+1}$. For this, the equation 3.2 is employed which can be approximated as

$$
h_{j}^{n+1}=h_{j}^{n}+\frac{\phi_{j}^{n+1}-\phi_{j}^{n}}{\bar{K}\left(h_{j}^{n}\right)} .
$$

\section{2. von Neumann Stability analysis. .}

From equation (3.6), the difference scheme for $\lambda=\frac{1}{2}$ can be written as

$-\frac{1}{2} \sigma_{j}^{n} \phi_{j-1}^{n+1}+\left(1+\sigma_{j}^{n}\right) \phi_{j}^{n+1}-\frac{1}{2} \sigma_{j}^{n} \phi_{j+1}^{n+1}=\frac{1}{2} \sigma_{j}^{n} \phi_{j-1}^{n}+\left[1-\sigma_{j}^{n}\right] \phi_{j}^{n}+\frac{1}{2} \sigma_{j}^{n} \phi_{j+1}^{n}+\sigma_{i j}^{n}(E T)_{j}^{n}, \quad j=0, \pm 1, \ldots$

at $(n+1)$ time step, the error calculation has in the form:

$-\frac{1}{2} \sigma_{j}^{n} E_{j-1}^{n+1}+\left(1+\sigma_{j}^{n}\right) E_{j}^{n+1}-\frac{1}{2} \sigma_{j}^{n} E_{j+1}^{n+1}=\frac{1}{2} \sigma_{j}^{n} E_{j-1}^{n}+\left[1-\sigma_{j}^{n}\right] E_{j}^{n}+\frac{1}{2} \sigma_{j}^{n} E_{j+1}^{n}, j=0, \pm 1, \ldots$

assuming a Fourier component for

$$
E_{j}^{n}=\tilde{E}^{n} e^{j i 2 \pi h \xi}
$$


The scheme 3.12 becomes:

$$
\begin{gathered}
-\frac{1}{2} \sigma_{j}^{n} e^{-2 i \pi h \xi} \tilde{E}^{n+1}+\left(1+\sigma_{j}^{n}\right) \tilde{E}^{n+1}-\frac{1}{2} \sigma_{j}^{n} e^{2 i \pi h \xi} \tilde{E}^{n+1}=\frac{1}{2} \sigma_{j}^{n} e^{-2 i \pi h \xi} \tilde{E}^{n}+\left[1-\sigma_{j}^{n}\right] \tilde{E}^{n}+\frac{1}{2} \sigma_{j}^{n} e^{2 i \pi h \xi} \tilde{E}^{n} \\
\Rightarrow \frac{1}{2} \sigma_{j}^{n}\left(-e^{-2 i \pi h \xi}+2-e^{2 i \pi h \xi}\right) E^{\tilde{n}+1}+2 E^{\tilde{n}+1}=\frac{1}{2} \sigma_{j}^{n}\left(e^{-2 i \pi h \xi}-2+e^{2 i \pi h \xi}\right) \tilde{E}^{n}+2 \tilde{\phi}^{n}
\end{gathered}
$$

Introducing an amplification factor such that $\tilde{E}_{j}^{n+1}=G \tilde{E}_{j}^{n}$, then

$$
\tilde{E}^{n+1}\left(1+\frac{1}{2} \sigma_{j}^{n}\left(-e^{-2 i \pi h \xi}+2-e^{2 i \pi h \xi}\right)\right)=\tilde{E}^{n}\left(1+\frac{1}{2} \sigma_{j}^{n}\left(e^{-2 i \pi h \xi}-2+e^{2 i \pi h \xi}\right)\right)
$$

Utilizing the relation

$$
\begin{gathered}
2 h \pi \xi=u, \cos u=\frac{e^{i u}+e^{-i u}}{2} \\
\tilde{E}^{n+1}\left(1+\sigma_{j}^{n}(1-\cos u)\right)=\tilde{E}^{n}\left(1-\sigma_{j}^{n}(1-\cos u)\right) \\
\Rightarrow G=\frac{\left(1-\sigma_{j}^{n}(1-\cos u)\right)}{\left(1+\sigma_{j}^{n}(1-\cos u)\right)}=\frac{1-2 \sigma_{j}^{n} \sin ^{2} \frac{u}{2}}{1+2 \sigma_{j}^{n} \sin ^{2} \frac{u}{2}}
\end{gathered}
$$

For a stable solution the absolute value of $\mathrm{G}$ must be less than or equal to 1 for all values of $u$. Since $G^{\prime}(u)=0$ at $u=0, \pm \pi$ and $G(0)=1$ the stability requirement can be expressed as

$$
\frac{\left(1-2 \sigma_{j}^{n}\right.}{1+2 \sigma_{j}^{n}} \leq 1
$$

is always true for

$$
\sigma_{j}^{n} \geq 0
$$

It can be shown that the numerical scheme (3.9) is unconditionally stable. Note that when $c(\phi)=0$ in $(3.3)$ becomes purely a diffusion equation. Thus, to avoid degeneracy it needs to adjust a small parameter in the calculation of $c\left(\phi_{j}^{n}\right)$.

\section{Simulation Results}

4.1. Numerical Setup. The numerical procedure developed in the previous section is written in Python, and ran on a laptop with $2.8 \mathrm{GHz}$ Quad-Core Intel Core i7 processor - We inspect the behavior of the numerical scheme presented in the previous section in a specific infiltration experiment. In this simulation we consider a vertical soil column of depth $L=70 \mathrm{~cm}$ in a time period of $t_{\max }=1 \mathrm{hr}$ with reference evapotranspiration .0000175 $\mathrm{cm} / \mathrm{hr}$.

Following Haverkamp et al. [8], using the soil parameters and the characteristics relationship between the soil moisture content $\theta(\psi)$ and the hydraulic conductivity $K(\psi)$ in the following way

$$
\begin{gathered}
\theta(\psi)=\theta_{r}+\frac{\alpha\left(\theta_{s}-\theta_{r}\right)}{\alpha+|\psi|^{\beta}}=0.075+\frac{1.616 \times 10^{6}(.287-.075)}{1.616 \times 10^{6}+|\psi|^{3.96}} \\
K(\psi)=K_{s} \frac{A}{A+|\psi|^{\gamma}}=\frac{34 \times 1.175 \times 10^{6}}{1.175 \times 10^{6}+|\psi|^{4.74}}
\end{gathered}
$$


The simulation starts with a uniform saturation $\theta=0.1 \mathrm{~cm}^{3} / \mathrm{cm}^{3}$ and a constant water head $\psi=-61.5 \mathrm{~cm}$ is maintained at the bottom boundary $z=L$. At the upper boundary $z=0$ (the soil surface), a constant flux $q(t)=13.69 \mathrm{~cm} / \mathrm{hr}$ for $t<0.3 \mathrm{hr}$ and zero normal flux condition for $t>0.3 \mathrm{hr}$.

The problem is simulate with Crank-Nicolson method which is unconditionally stable and have no restriction of time step size. The step size is limited only by the accuracy of approximation. We run $\mathrm{CN}$ scheme for the problem with or without sink terms and compare the results. To deal with the nonlinear dependence of the functional coefficient $c(\phi)$ in the $\mathrm{CN}$ scheme, we use fix point iteration with maximum allowable iteration $M A X I T=10$ and relative error tolerance $T O L=10^{-6}$.

4.2. Results and Discussion. Richards equation is highly non-linear. The non-linear behavior of Richards equation appears by use of relationship between $\theta$ and $\psi$ and $K$ and $\psi$. Figure 1 shows the relative curve developed by Haverkamp et al. [8] using experimental data with appropriate parameter values.
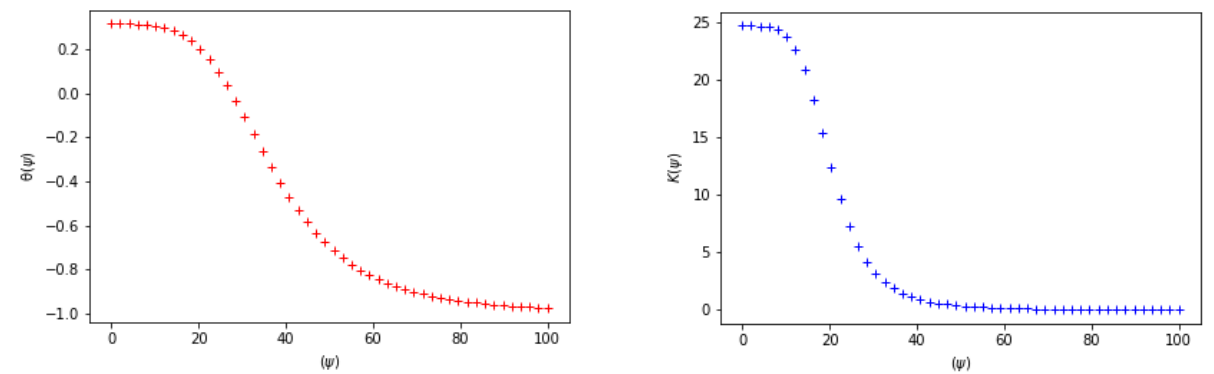

Figure 1. The shape of function $\theta(\psi)$ (left) and $K(\psi)$ (right).
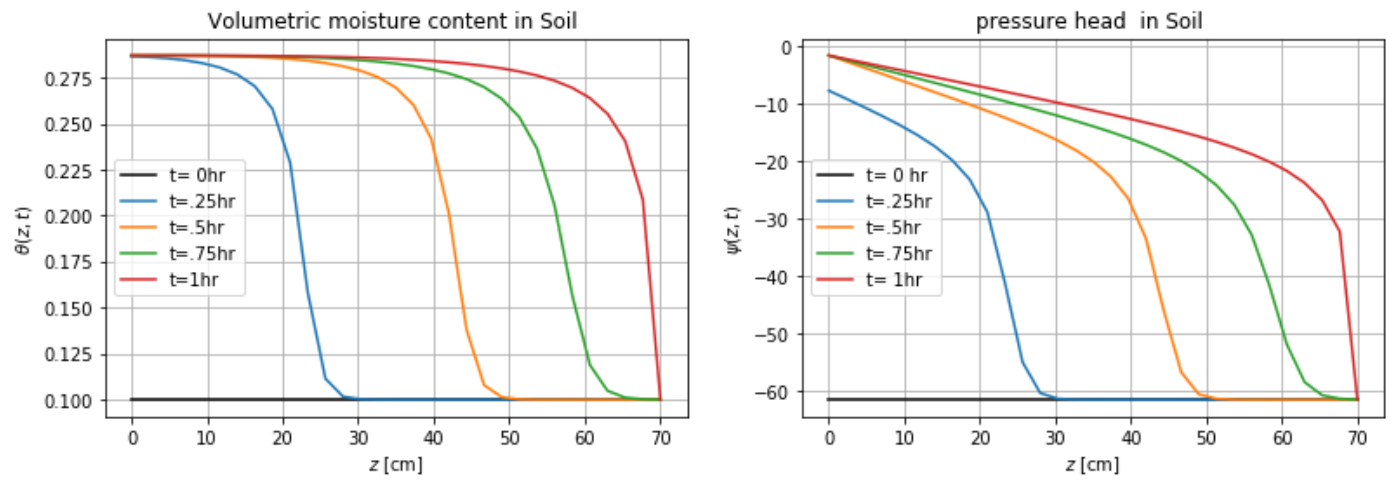

FigURE 2. variational trend of moisture content in depth(left) and pressure head(right) without evapotranspiration.

The numerical solution of Richards equation has computed using Crank-Nicolson method - First it has computed the equation without evapotranspiration with a fairly fine mesh of $\Delta z=2 \mathrm{~cm}$ and $\Delta t=0.001 \mathrm{sec}$ as the reference solution. Figure 2 describes the profile of moisture retention function and the pressure head phenomenon. In which the drainage 

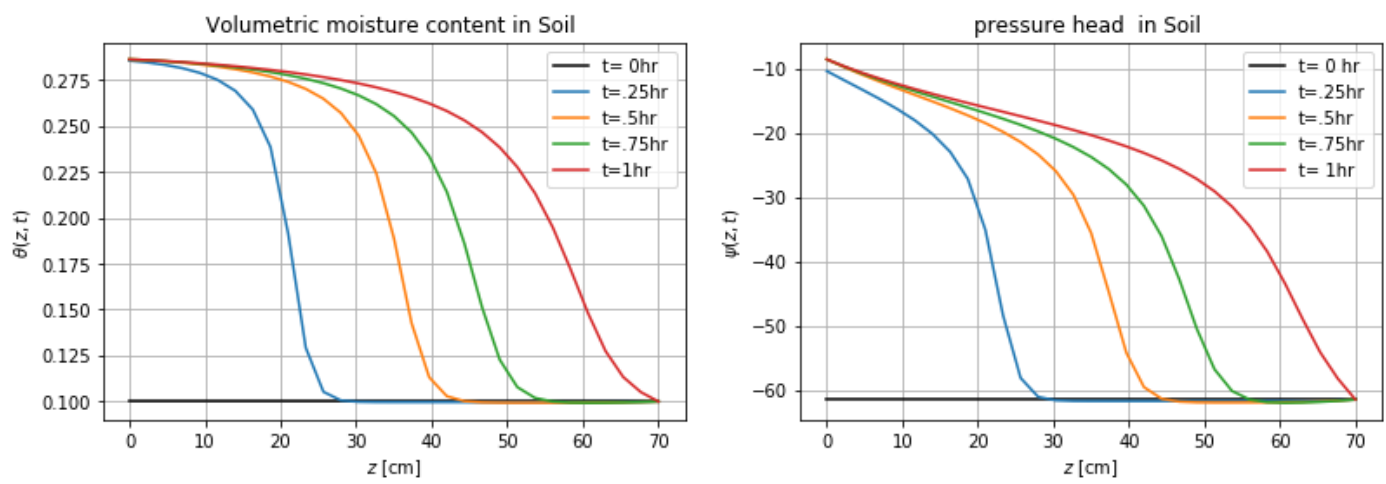

FiguRE 3. variational trend of moisture content in depth(left) and pressure head(right) with evapotranspiration.

takes place at $29 \mathrm{~cm}, 50 \mathrm{~cm}$, and $65 \mathrm{~cm}$ at the corresponding time $.25 \mathrm{hr}, .50 \mathrm{hr}, .75 \mathrm{hr}$ respectively. Secondly the equation has computed with evapotranspiration as the same mesh and same time step with reference evapotranspiration $0.0000175 \mathrm{~cm} / \mathrm{hr}$. Figure 3 describes the corresponding profile for pressure head and the moisturity. Where the drainage takes place at $26 \mathrm{~cm}, 42 \mathrm{~cm}$, and $55 \mathrm{~cm}$ at the corresponding time $.25 \mathrm{hr}, .50 \mathrm{hr}, .75 \mathrm{hr}$ respectively. The comparison between the computed result shows that the cost for computation in drainage is expensive in the case of Richards equation without the loss of water due to the evaporation of unsaturated porous media (soils) and transpiration of plants. in figure 4 and 5 , we describe the profile of moisture content $\theta(z, t)$ and pressure head $\psi(z, t)$ along the soil depth at various times $t=0.1,0.2, \ldots, 1 h r$ without evapotranspiration and with evaporation.
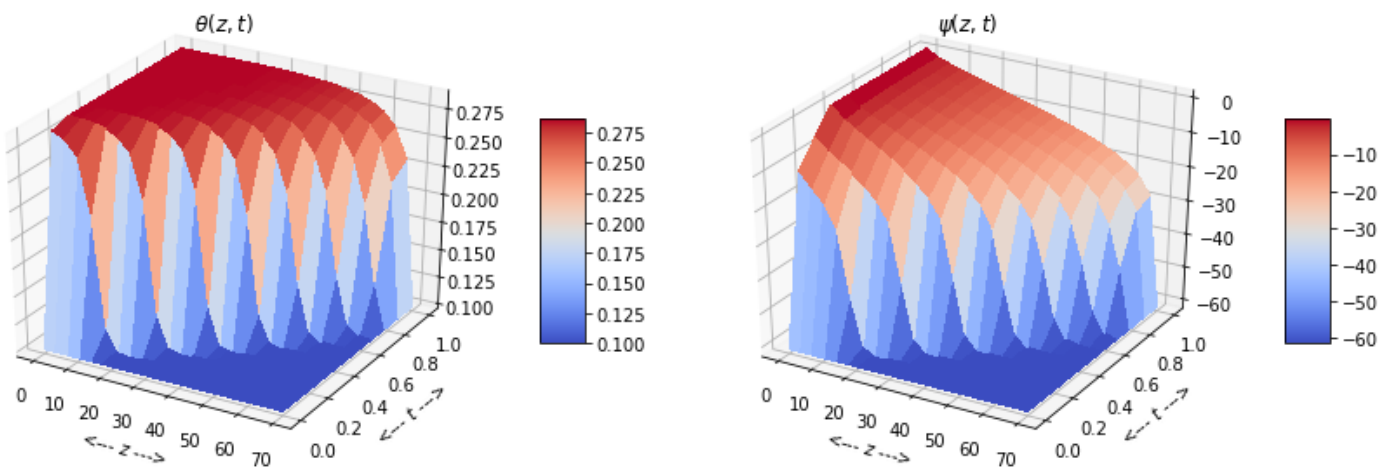

FiguRE 4. Variational trend of moisture content in depth and time (left) and pressure head (right) without evapotranspiration

\section{Conclusion}

In this work, we considered one-dimensional Richards equation and solve it numerically by using Crank-Nicolson method. We compare the model with and without the loss of water due to the evaporation of unsaturated porous media (soils) and transpiration of plants. Our result has realistic significance as expected. It has shown that an additional work in time 

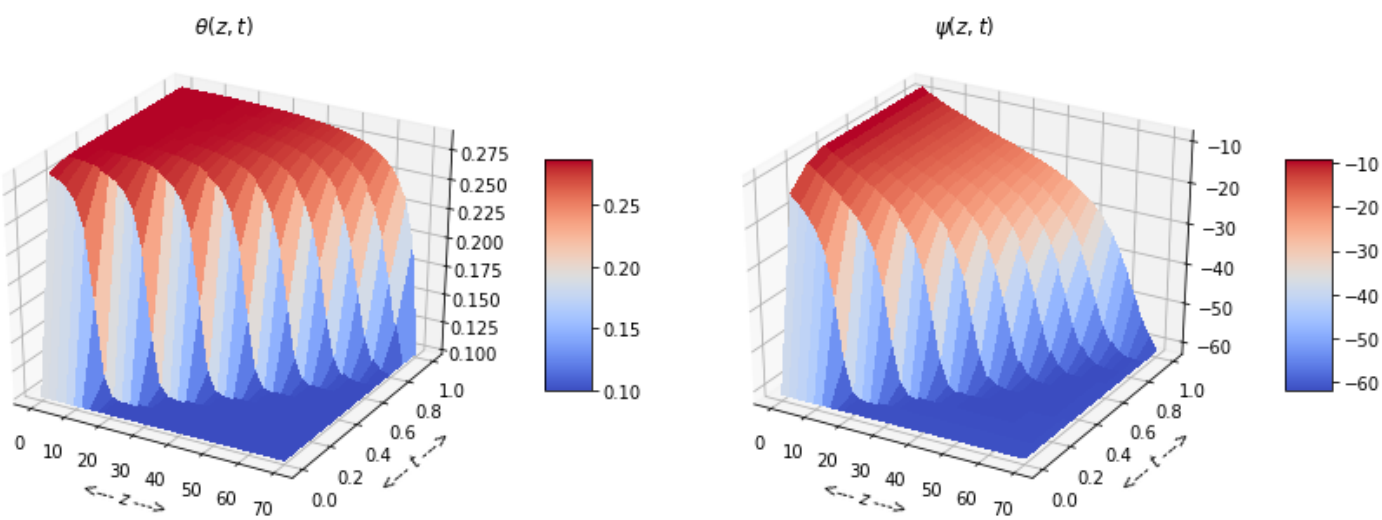

FiguRE 5. Variational trend of moisture content in depth and time (left) and pressure head (right) with evapotranspiration

and flow movement is needs to reach the given boundary condition for the model without evapotranspiration. Although Richards equation is a phenomenological model, depending on Darcy's law and constitutive relationship $\theta(\psi)$ and $K(\psi)$, equation (1.1) becomes a useful model with the ability to quantitatively predict water infiltration in unsaturated soil with naturally recharge and evapotranspiration for higher dimension.

Data Availability

The data used for supporting the findings of this study are included within the article.

\section{Conflicts of Interest}

The authors declare that there are no conflict of interest.

\section{REFERENCES}

[1] Richards, L. A., "Capillary conduction of liquids through porous mediums",Physics 1, (5), 318-333. 1931.

[2] Van Genuchten, M.Th., E.A. Sudicky, "Recent Advances in Vadose Zone flow and transport modeling, Vadose Zone Hydrology cutting across disciplines", Oxford University Press, 155-193, 1999.

[3] R.G. Allen, L.S Pereira,D.Raes, and M. Smith, "Crop Evapotranspiration-guidelines for computing Crop water Requirements-FAO",Irigation and Drainage paper 56,vol.300 FAO,Rome, 1998.

[4] Celia, M. A. and E. T. Bouloutas, "A General Mass-Conservative Numerical solution for the Unsaturated flow equation", Water Resource research, 26, 1483-1490, 1990.

[5] Berganaschi, L., PUTTI, M., "Mixed finite elements and Newton-type militarizations for the solution of the Richards equations", Int.J. Numer. Methods Eng., 45, 1025-1046, 1999.

[6] Eymard, R., Gutnic, M., Hillhorst, D., "The finite volume method for richard equation", textitCompute. Geosci, 3, 259-294, 1999.

[7] Gottardi, G. and M. Venuteli, "Richards: Computer Programming for the numerical simulation of one-dimensional infiltration into unsaturated soil",Computers and Geo-sciences, 19, 1239-1266, 1993.

[8] Haverkamp, R., M. Vauclin, J. Touma, P. J. Wierenga, G. Vachaud, "A comparison of numerical simulation models for one-dimensional infiltration", Soil Sci. Soc. Am. J., 41(2),285-294, 1997.

[9] Liu, F., Fukumoto, Y., Zhao, X., "A linearized finite difference Scheme for the Richards equation under variable-Flux boundary conditions", Journal of scientific computing, 83, 2-21, 2020.

[10] Zambra,C.E., Dumbser, M., Toro , E.F. and Morago, N.O., " A novel numerical method of high-order accuracy for flow in unsaturated porous media". Int. J. Numer. Meth. Engng, 89, 227-240,2012. 
[11] Kavetsky, D., Binning, P., Sloan, S.W., " Adaptive time stepping and error control in a mass conservative numerical solution of the mixed form of Richards equation", Adv. Water Res., 24, 595-605, 2001.

[12] Radu, F. A. and Florian List, "A Study on iterative methods for solving Richards equation", Computers and Geo-sciences, 20,341-353, 2016.

[13] Egid,N., E. Gioia, P. Maponi and L. Spadoni., "A numerical solution of Richards equation: a simple method adaptable in parallel computing", International Journal of computer Mathematics, 97, 2-17, 2020 .

[14] Timsina R.C, Khanal H., Uprety K.N,(2021) "An explicit stabilized Runge-Kutta-Legendre super time -stepping scheme for the Richards equation", Mathematical problems in Engineering, vol-2021, 1-11, 2021

[15] J.W. Thomas, "Numerical partial differential equations: Finite difference methods", Text in Applied Mathematics, Springer, 2010. 